\title{
First results of the Southern Open Cluster Study
}

\author{
K. Kinemuchi, ${ }^{1,2}$ A. Sarajedini, ${ }^{2}$ D. Geisler,${ }^{1}$ F. Mauro, ${ }^{1}$ G. Carraro,${ }^{3}$ \\ S. Kafka ${ }^{4}$ and E. Jeffery ${ }^{5}$ \\ ${ }^{1}$ Departamento de Astronomía, Universidad de Concepción, Concepción, Chile \\ email:[kkinemuchi, dgeisler, fmauro] @astro-udec.cl \\ ${ }^{2}$ Department of Astronomy, University of Florida, 211 Bryant Space Science Center, \\ Gainesville, FL 32611, USA \\ email: [kinemuchi, ata] @astro.ufl.edu \\ ${ }^{3}$ European Southern Observatory \\ email: gcarraro@eso.org \\ ${ }^{4}$ SSC/Caltech, Pasadena, CA 91125, USA \\ email:stella@caltech.edu \\ ${ }^{5}$ Department of Astronomy, University of Texas at Austin, Austin, TX 78712, USA \\ email: ejeffery@astro.as.utexas.edu
}

\begin{abstract}
Galactic open clusters are excellent laboratories to study stellar populations that are coeval and have a common chemical composition. These clusters allow for the investigation of stellar and chemical abundance evolution, binary star systems, mass and luminosity functions, Galactic metallicity gradient, and disk structure, among other things. The Southern Open Cluster Study (SOCS) is a database of a select set of 24 open clusters with a broad range of ages and metallicities. It is an extension of the northern WIYN Open Cluster Study (wocs). The main goal of both studies is to obtain comprehensive photometric, spectroscopic, and astrometric information of these key open clusters. Here, we provide an overview of the socs survey and present results of the wide-field photometry on two socs clusters, NGC 3532 and Tombaugh 2.
\end{abstract}

Keywords. surveys, open clusters and associations: individual (NGC 3532, Tombaugh 2)

\section{Introduction}

The Southern Open Cluster Study (SOCs) is a comprehensive study of 24 carefully selected southern open clusters. This survey is the southern extension of the northern survey, the WIYN Open Cluster Study (wocs; Mathieu 2000; Sarajedini et al. 2003). The socs survey has similar science goals as the wOCs study, primarily to provide a complete and detailed analysis of the clusters through photometry and spectroscopy. This database will be an excellent resource for other studies related to open clusters and their stellar populations.

The primary goals of the socs survey can be described by three aspects: photometry, spectroscopy, and astrometry. Presently, our photometric campaign is nearly complete. We have achieved both wide-field and deep pencil-beam photometry for color-magnitude diagrams in a variety of optical bandpasses. For the spectroscopy of the socs clusters, we will obtain chemical abundances and stellar radial velocities. Combining all available kinematic resources, we will determine precise positions and proper-motion membership of the cluster stars. We will improve the astrometric information for all our socs clusters.

The open clusters selected for socs have a wide range in age (0.04-8.9 Gyr) and metallicity $(-0.5<[\mathrm{Fe} / \mathrm{H}]<+0.1)$. A list of the socs clusters is presented in Table 1 . 
Table 1. socs clusters and their parameters.

\begin{tabular}{lllrll}
\hline Name & $\begin{array}{l}\text { RA } \\
(2000)\end{array}$ & $\begin{array}{l}\text { Dec } \\
(2000)\end{array}$ & $\begin{array}{r}(m-M) \\
(\mathrm{mag})\end{array}$ & $\begin{array}{l}{[\mathrm{Fe} / \mathrm{H}]} \\
(\mathrm{dex})\end{array}$ & $\begin{array}{l}\text { Age } \\
(\mathrm{Gyr})\end{array}$ \\
\hline NGC 2204 & $06: 15: 33$ & $-18: 39: 54$ & 12.4 & -0.3 & 0.8 \\
NGC 2243 & $06: 29: 34$ & $-31: 17: 00$ & 13.4 & -0.4 & 4.5 \\
NGC 2287 & $06: 46: 01$ & $-20: 45: 24$ & 9.3 & +0.04 & 1.9 \\
NGC 2360 & $07: 17: 43$ & $-15: 38: 30$ & 11.7 & -0.2 & 0.6 \\
Tombaugh 2 & $07: 03: 05$ & $-20: 49: 00$ & 15.9 & -0.4 & 1.0 \\
Melotte 66 & $07: 26: 23$ & $-47: 40: 00$ & 13.6 & -0.4 & 2.8 \\
NGC 2423 & $07: 37: 06$ & $-13: 52: 18$ & $\ldots$ & $\ldots$ & $\ldots$ \\
NGC 2451 & $07: 44: 27$ & $-37: 40: 00$ & 7.6 & -0.5 & 0.04 \\
Berkeley 39 & $07: 46: 42$ & $-04: 36: 00$ & 13.8 & -0.2 & 7.9 \\
NGC 2477 & $07: 52: 10$ & $-38: 31: 48$ & 11.3 & +0.01 & 0.7 \\
NGC 2516 & $07: 58: 04$ & $-60: 45: 12$ & 8.4 & +0.06 & 0.1 \\
NGC 2506 & $08: 00: 01$ & $-10: 46: 12$ & 13.0 & -0.4 & 1.1 \\
NGC 2547 & $08: 10: 09$ & $-49: 12: 54$ & 8.4 & -0.16 & 0.36 \\
NGC 2539 & $08: 10: 37$ & $-12: 49: 06$ & 10.9 & +0.13 & 0.37 \\
NGC 2546 & $08: 12: 15$ & $-37: 34: 42$ & 10.2 & +0.12 & 0.74 \\
IC 2395 & $08: 42: 30$ & $-48: 09: 00$ & 9.5 & $\ldots$ & 0.16 \\
NGC 2660 & $08: 42: 38$ & $-47: 12: 00$ & 13.2 & -0.2 & 1.1 \\
NGC 3532 & $11: 05: 39$ & $-58: 45: 12$ & 8.6 & -0.02 & 0.3 \\
NGC 3680 & $11: 25: 38$ & $-43: 14: 36$ & 10.1 & -0.1 & 1.2 \\
NGC 3960 & $11: 50: 33$ & $-55: 42: 24$ & 12.7 & -0.2 & 0.7 \\
NGC 4103 & $12: 06: 40$ & $-61: 15: 00$ & 12.0 & $\ldots$ & 0.27 \\
CR 261 & $12: 37: 57$ & $-68: 22: 00$ & 12.5 & -0.1 & 8.9 \\
NGC 5822 & $15: 04: 21$ & $-54: 23: 48$ & 10.3 & -0.02 & 0.7 \\
IC 4651 & $17: 24: 49$ & $-49: 56: 00$ & 10.1 & +0.1 & 1.1 \\
\hline
\end{tabular}

To demonstrate the spatial distribution of the open clusters, Figure 1 shows where the clusters are located in an Aitoff plot with Galactic coordinates. Figure 2 shows the metallicity distribution of the socs clusters with respect to $\log ($ age $)$.

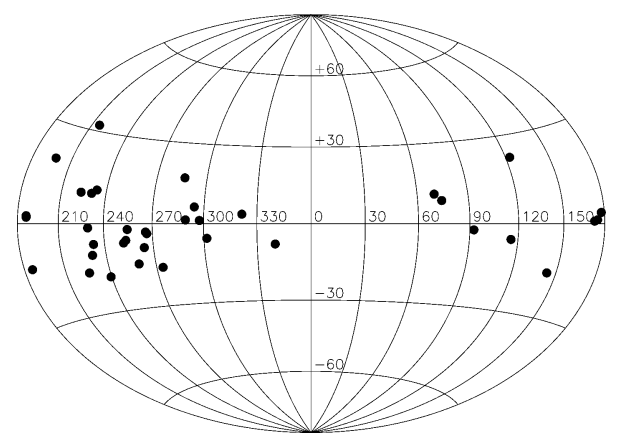

Figure 1. Aitoff plot in Galactic coordinates of the wocs/socs clusters.

\section{Data acquisition for photometry}

For the wide-field photometry, all data were obtained at the Warsaw 1.3m telescope at Las Campanas Observatory and the $1.0 \mathrm{~m}$ telescope at Cerro Tololo Inter-American Observatory (CTIO), Chile. The open clusters were observed in 2005 and 2007 during four separate observing runs. The Warsaw telescope had a MOSAIC CCD camera with a field of view of $34^{\prime} \times 34^{\prime}$. The open clusters were observed in the Johnson $B V R I$ filters 


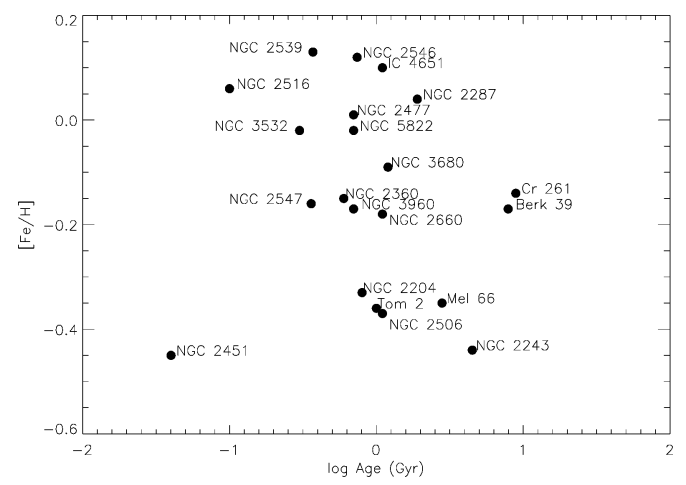

Figure 2. Distribution of the SOCs clusters with respect to metallicity and $\log ($ age).

with three different exposure times. Six open clusters were observed in $U B V R I$ filters at the CTIO $1.0 \mathrm{~m}$, which covered a smaller region $\left(20^{\prime} \times 20^{\prime}\right)$.

To perform point-spread-function photometry on the images, we developed a pipeline which interfaced with the stand-alone version of DAOPнот IV (Stetson 1987, 1994). All data from the Warsaw $1.3 \mathrm{~m}$ telescope were processed and each open cluster is being systematically photometered. In these proceedings, we present our preliminary results of two socs clusters from our photometric campaign.

\section{Wide-field photometry results}

We present color-magnitude diagrams (CMDs) of the socs open clusters NGC 3532 and Tombaugh 2. We provide our results with a preliminary photometric calibration of the CMDs. Further work is underway to determine the final standard calibration and to perform a careful analysis of the stellar populations.

\section{1. $N G C 3532$}

For this relatively metal-rich open cluster, we present in Figure 3 the CMD. Even with the strong field contamination, we can clearly see the cluster's main sequence and a hint of the turnoff. Data for this CMD was culled primarily from the inner three MOSAIC chips of CCD camera at the Warsaw telescope.

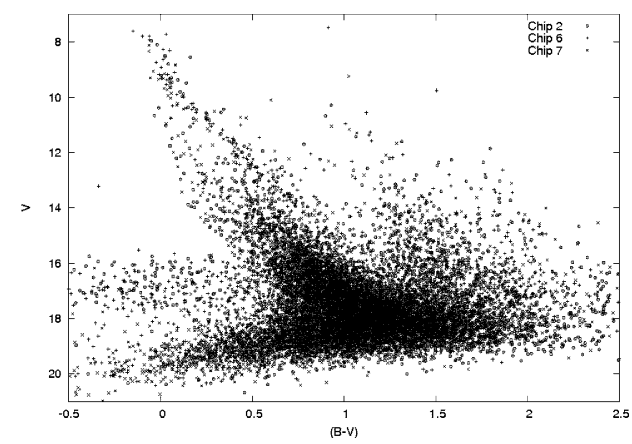

Figure 3. Color-magnitude diagram of NGC 3532.

(Data from the three inner MOSAIC chips.) 


\subsection{Tombaugh 2}

Data for Tombaugh 2 were also obtained at the Warsaw $1.3 \mathrm{~m}$ in 2007. The CMD in Figure 4 is also done with a preliminary calibration, but the diagram is composed of data from all eight chips of the MOSAIC camera. There is still heavy field contamination. However, one can see the main-sequence turnoff and the stars associated with the cluster's red clump. Standard calibration and analysis of Tombaugh 2 is currently underway.

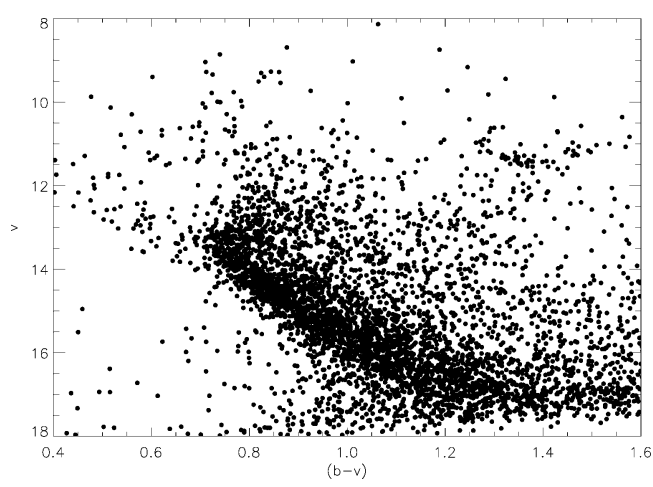

Figure 4. CMD of Tombaugh 2.

\section{Future work}

The socs project is now finishing up the optical-photometry portion but has delved into near-infrared (NIR) photometry. We have NIR data for two socs clusters taken with the ISPI instrument at the CTIO Blanco 4.0m telescope. The NIR data will be particularly useful in understanding the binary-star population in these open clusters (see Robitaille et al. 2007; Frinchaboy \& Nielsen 2008). Projects are planned for the spectroscopy of the socs clusters, mainly to investigate their chemical abundances in detail.

\section{Acknowledgements}

KK, AS, and DG gratefully acknowledge support from the National Science Foundation through grant AST-0606703.

\section{References}

Frinchaboy, P. M. \& Nielsen, D. 2008, in: E. Vesperini, M. Giersz \& A. Sills (eds.), Dynamical Evolution of Dense Stellar Systems, Proc. IAU Symp. 246, p. 109

Mathieu, R. D. 2000, in: R. Pallavicini, G. Micela, \& S. Sciortino (eds.), Stellar Clusters and Associations: Convection, Rotation, and Dynamos, ASP Conf. Ser., 198, p. 517, San Francisco: ASP

Robitaille, T. P., Whitney, B. A., Indebetouw, R., \& Wood, K. 2007, ApJS, 169, 328

Sarajedini, A., Mathieu, R. D., \& Platais, I. 2003, in: T. D. Oswalt (ed.), The Future of Small Telescopes In The New Millennium. III. Science in the Shadows of Giants, ASSL, 289, 257

Stetson, P. B. 1987, PASP, 99, 191

Stetson, P. B. 1994, PASP, 106, 250 